\title{
Können Bisphosphonate die Prognose von Mammakarzinom- Patientinnen mit DTCs im Knochenmark verbessern?
}

Heute geht man davon aus, dass die Disseminierung von Tumorzellen bei Mammakarzinom-Patientinnen bereits in sehr frühen Erkrankungsstadien erfolgen kann. Die disseminierten Tumorzellen (DTCs) können mehrere Jahre nach der Primärtherapie eines Mammakarzinoms im Knochenmark persistieren und gelten als unabhängiger prognostischer Faktor für das metastasenfreie und brustkrebsspezifische Überleben. Im fortgeschrittenen Erkrankungsstadium reduziert eine systemische Therapie mit Bisphosphonaten bei Mammakarzinom-Patientinnen mit Knochenmetastasen das Risiko skelettbezogener Komplikationen (skeletal-related events (SREs)). Aber auch in der adjuvanten Situation spielen Bisphosphonate wie das orale Clodronat bei der Prophylaxe von Metastasen bei Patientinnen mit nachgewiesenen DTCs im Knochenmark eine wichtige Rolle. Eine Reihe von Studien belegt den Nutzen einer adjuvanten Bisphosphonat-Therapie zur Prävention von Knochenmetastasen und zur Verbesserung des brustkrebsspezifischen Überlebens bei ausgewählten Patientengruppen.

In vielen Fällen kommt es beim Mammakarzinom zu einer frühzeitigen okkulten Disseminierung von Tumorzellen. Befinden sich die Tumorzellen im Blut- und Lymphsystem, spricht man von zirkulierenden Tumorzellen (CTCs). Gelangen sie ins Knochenmark, können sie dort über mehrere Jahre verbleiben und stellen als solche die hauptsächliche Form der disseminierten Tumorzellen (DTCs) dar. Sie sind metabolisch inaktiv und werden auch als «sleeper cells» oder «dormant cells» bezeichnet [1]. Erst wenn sich DTCs erneut an Geweben anlagern und proliferieren, spricht man von Metastasen.

Im Knochenmark lassen sich DTCs über Antikörper-basierte immunologische Methoden (Durchflusszytometrie, Immunhistologie) und spezifische Marker nachweisen. Der immunzytochemische Nachweis Zytokeratin (CK)-positiver Zellen gilt als das momentan sensitivste und am besten validierte Standardverfahren zum Nachweis einer okkulten Frühdisseminierung von Tumorzellen. Durch noch nicht bekannte Signale können die DTCs erwachen und sich vom Knochenmark ausgehend weiter systemisch verteilen $[2,3]$.

\section{DTCs im Knochenmark verschlechtern die Prognose von Mammakarzinom-Patientinnen}

In einer Reihe von klinischen Studien wurde die Aussagekraft von DTCs als Prognosefaktor für das progressionsfreie Überleben (PFS) und Gesamtüberleben (OS) bei Frauen mit Mammakarzinom untersucht [4-6]. Die Studien zeigten, dass das Vorhandensein von DTCs mit einem reduzierten rezidivfreien Überleben und dem Auftreten von Fernmetastasen und krebsbedingten Todesfällen korrelierte und einen unabhängigen negativen Prognosefaktor für die krebsassoziierte Mortalität darstellt. Auch eine Untersuchung von Diel et al. [7] ergab, dass Mammakarzinom-Patientinnen eine schlechtere Prognose haben, wenn bei ihnen DTCs im Knochenmark nachgewiesen werden konnten. Eine große Metaanalyse untermauerte die signifikante prognostische Aussagekraft für das rezidiv- und metastasenfreie Überleben sowie das OS [8].

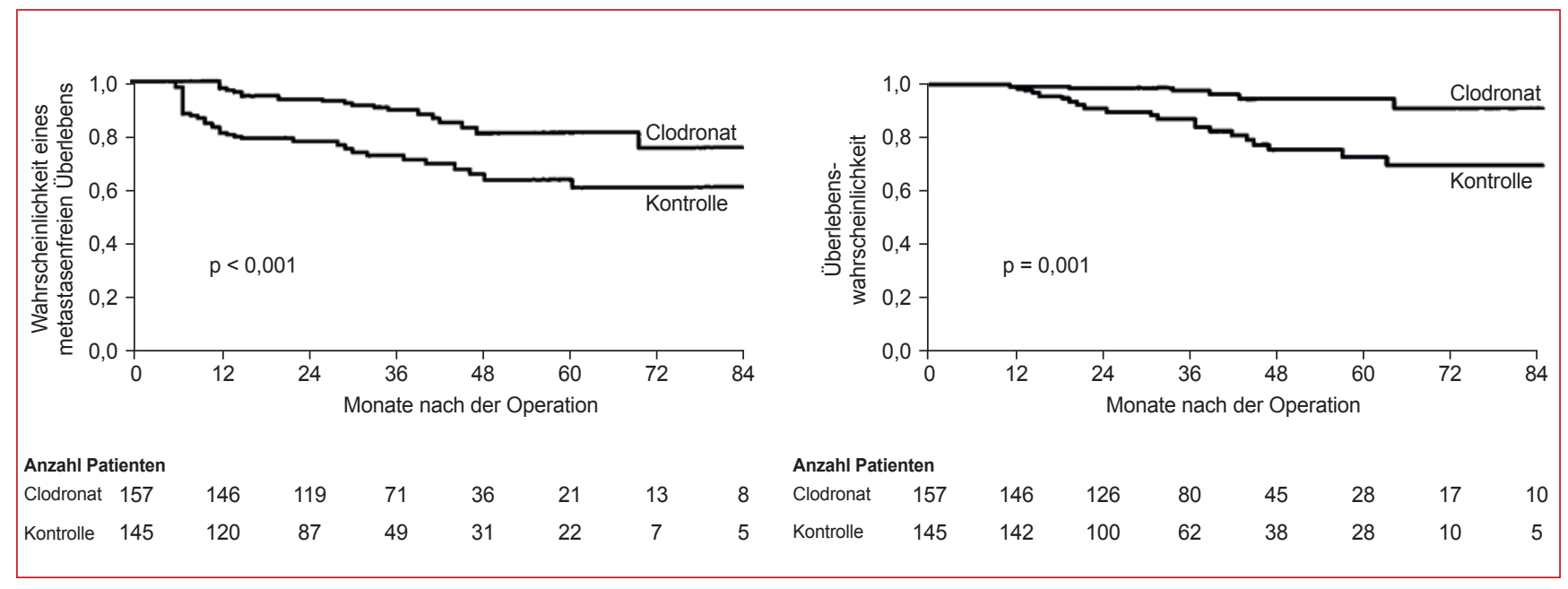

Abb. 1. Signifikante Verlängerung des metastasenfreien Überlebens und Gesamtüberlebens bei den mit Clodronat behandelten Patientinnen; modifiziert nach [9].

S. Karger

Medical and Scientific Publishers

Basel · Freiburg · Paris - London .

New York · Chennai · New Delhi

Bangkok · Beijing · Shanghai .

Tokyo - Kuala Lumpur .

Singapore · Sydney
Beilage zu 


\section{Studien belegen: Patientinnen mit DTCs und post- menopausale Frauen profitieren von adjuvantem Clodronat}

Verschiedene Studien haben den adjuvanten Einsatz von Bisphosphonaten bei Mammakarzinom-Patientinnen untersucht. So zeigte die erste publizierte prospektive Studie zur Metastasenprophylaxe mit Bisphosphonaten bei 302 Mammakarzinom-Patientinnen mit nachgewiesenen DTCs, dass 1600 mg Clodronat täglich das Auftreten von ossären und auch viszeralen Metastasen signifikant reduziert $(p<0,001)$ und das Überleben verbessert [9] ( $p=0,001$; Abb. 1). In diese Studie wurden ausschließlich Hochrisiko-Patientinnen mit nachgewiesenen DTCs eingeschlossen. Bei einer Nachuntersuchung nach 8,5 Jahren zeigte sich immer noch die signifikante Verbesserung des OS durch Clodronat [10].

Vier Jahre später zeigten Powles et al. [11] in einer Studie zur Prävention von Knochenmetastasen bei 1069 Patientinnen mit primärem Mammakarzinom, dass $1600 \mathrm{mg}$ Clodronat täglich die Mortalität der Patientinnen im Stadium II und III signifikant reduziert. In der Placebogruppe verstarben 129 Patientinnen $(23,9 \%)$ im Vergleich zu 98 in der Clodronat-Gruppe (18,5\%). Dies entspricht einer Risikoreduktion um 23\% ( $\mathrm{p}=0,048)$.

Die NSABP-34-Studie ist mit 3323 eingeschlossenen Patientinnen die bisher größte Studie, die den Einfluss von adjuvantem Clodronat auf das krankheitsfreie Überleben (DFS) untersuchte [12]. Zwei Drittel der Patientinnen waren 50 Jahre oder älter. Sie erhielten eine adjuvante Chemo- und/oder endokrine Therapie. Das mediane Follow-up betrug 90,7 Monate. Zunächst ergab sich kein deutlicher Vorteil für den Einsatz von Clodronat bezogen auf das DFS, das knochenmetastasen- und rezidivfreie Überleben sowie das OS. Jedoch zeigte die Subgruppenanalyse bei postmenopausalen Patientinnen eine 25\%ige Verbesserung beim rezidivfreien Überleben.

Auf dem San Antonio Breast Cancer Symposium 2013 stellten Coleman et al. [13] die EBCTCG-Metaanalyse zur adjuvanten Bisphosphonattherapie zur Vermeidung von Metastasen beim Mammakarzinom vor. Alle Frauen über 55 Jahre mit unbekanntem Menopausenstatus wurden als postmenopausal klassifiziert. Nach 10 Jahren Nachbeobachtungszeit zeigte sich bei postmenopausalen Frauen eine signifikante Reduktion von Knochenmetastasen $(p=0,00001)$ und der brustkrebsspezifischen Mortalität $(p=0,004)$. Der Effekt war unabhängig vom Östrogenrezeptor- und Nodalstatus sowie dem Einsatz einer Chemotherapie. Die positiven Effekte wurden für Clodronat und Aminobisphosphonate in gleicher Weise nachgewiesen.

Als einzige negative Studie zeigt die Untersuchung von Saarto et al. [14] keine Verlängerung des OS und keine Reduktion von Knochenmetastasen, allerdings wurden bei der Studie methodische Mängel festgestellt.

\section{Retrospektive Analyse zeigt: \\ Frühzeitige Bisphosphonat-Einnahme verbessert das Überleben von DTC-positiven Patientinnen und nivelliert den negativen prognostischen Effekt}

Im Rahmen einer kürzlich publizierten, am Universitätsklinikum Tübingen durchgeführten großen Studie wurden
3141 Patientinnen mit frühem Mammakarzinom während der primären Operation Knochenmarksaspirate entnommen und auf DTCs untersucht [6]. Bei den 803 Patientinnen mit DTC-Nachweis analysierte man retrospektiv die prognostische Relevanz der DTCs und der adjuvanten Bisphosphonattherapie für das DFS und das OS. Das Vorhandensein von DTCs erwies sich als unabhängiger negativer Prognosefaktor sowohl für das DFS als auch für das OS. Bei DTC-positiven Patientinnen korrelierte die adjuvante Gabe von Bisphosphonaten signifikant mit einem verlängerten DFS ( $p<0,001, A b b .2)$ und OS $(\mathrm{p}=0,006)$.

Auch am Universitätsklinikum Essen werden allen dort behandelten Brustkrebspatientinnen während der operativen Entfernung des Primärtumors Knochenmarksaspirate entnommen und dann auf DTCs untersucht. Alle Patientinnen, bei denen DTCs im Knochenmark nachweisbar sind, erhalten dann aufgrund der von Diel et al. 1998 [9] gezeigten signifikanten Verbesserung des OS die Therapieempfehlung zur Einnahme von oralem Clodronat über 2 Jahre unabhängig vom Menopausenstatus.

Ein auf dem San Antonio Breast Cancer Symposium 2014 vorgestelltes Poster zeigte die Survival-Daten von 525 am Universitätsklinikum Essen behandelten Patientinnen mit oder ohne Nachweis von DTCs [15]. Von den 525 Patientinnen waren 211 (40\%) DTC-positiv, darunter viele mit pT1Tumoren sowie nodalnegative und prämenopausale Patientinnen. Patientinnen mit positivem DTC-Status erhielten 1040 mg Clodronat täglich. Nach einem medianen Followup von 58,6 Monaten betrug die OS-Rate $92 \%$. Trotz der schlechteren Prognosedaten der DTC-positiven Patientinnen zeigte sich sowohl bezüglich des PFS als auch des OS zwischen den beiden Gruppen kein Unterschied. Dieses Ergebnis sei auf die Tatsache zurückzuführen, dass die beobachteten Patientinnen beim Nachweis von DTCs im Knochenmark frühzeitig mit der Einnahme von Clodronat begonnen hätten, so die Autoren.

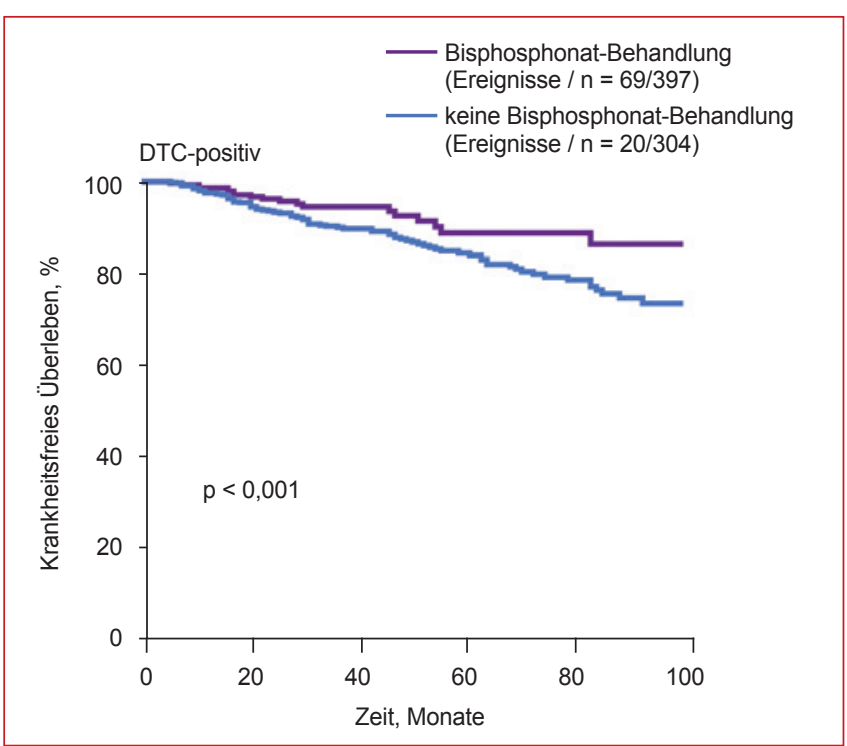

Abb. 2. Signifikant verlängertes DFS bei DTC-positiven Patientinnen, die eine adjuvante Bisphosphonattherapie erhalten haben; modifiziert nach [6]. 


\section{Interview mit Professor Ingo Diel zum Einfluss von Clodronat auf das Überleben von Patientinnen mit frühem Mammakarzinom und DTCs}

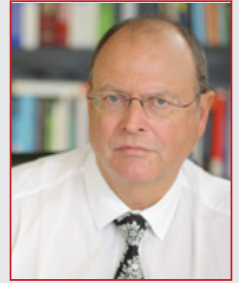

Prof. Dr. Ingo Diel, Facharzt für Gynäkologie und Geburtshilfe mit dem Schwerpunkt Gynäkologische Onkologie, Osteoonkologe, Mannheim

Was versteht man unter DTCs im Unterschied zu CTCs?

CTCs sind zirkulierende Tumorzellen, die im Blut nachgewiesen werden können und die sich noch nicht in Organen oder Geweben angesiedelt haben. DTCs sind Tumorzellen, die bereits ihren Zielort gefunden haben, nämlich das Knochenmark, wo man die Zellen dann auch nachweisen kann.

Welche Erklärung gibt es für die frühe Tumorzellabsiedelung beim Mammakarzinom?

Ein typisches Kennzeichen bösartigen Wachstums ist die Fähigkeit, an anderen Stellen des Körpers Metastasen abzusiedeln. Das gelingt dem Tumor nur dadurch, dass er Zellen in die Blutbahn abgibt, die dann im Körper verteilt werden und insbesondere im Knochenmark über längere Zeit verweilen können. Das ist für das Mammakarzinom und auch andere Tumoren, die in den Knochen metastasieren, sehr häufig nachzuweisen. Typischerweise läuft dieser Prozess schon in einem sehr frühen Stadium ab, wenn der Primärtumor noch nicht apparent ist.

Welche Bedeutung haben DTCs für die Prognose einer Patientin?

Meiner Ansicht nach bedeuten Tumorzellen im Knochenmark, ähnlich wie Tumorzellen in den Lymphknoten, dass der Tumor dazu in der Lage ist, Metastasen zu bilden. Die Tumorzellen im Knochenmark stellen ein Reservoir für spätere Metastasen dar. Man vermutet, dass es sich insbesondere um sogenannte Tumorstammzellen handelt, die die Fähigkeit haben, in einer fremden Umgebung länger zu überleben.

Und was bedeutet das für die Prognose?

Das bedeutet, dass das Risiko der Patientinnen für eine Metastasierung sehr viel höher liegt im Vergleich zu Frauen, bei denen man keine DTCs nachweisen kann.

Sind sie als unabhängiger Prognosefaktor anzusehen und, wenn, ja warum?

Nach den Berechnungen, die wir in Heidelberg an über 1000 Patientinnen durchgeführt haben, und aus anderen Untersuchungen mit großen Patientenzahlen wissen wir, dass die DTCs einen unabhängigen Prognosefaktor darstellen, der nicht mit dem Lymphknotenstatus korreliert. Dies erklärt, warum es immer wieder Frauen mit Mammakarzinom gibt, die zwar keine befallenen Lymphknoten haben, aber trotzdem Metastasen entwickeln.

Wie viele Patientinnen haben zum Zeitpunkt der Diagnose DTCs?

Beim Mammakarzinom sind es grob geschätzt $30 \%$. Es gibt Untersuchungen, die einen höheren Prozentsatz, und andere, die einen geringeren zeigen. Das hängt u.a. von der Nachweismethode ab. Die Methoden des Tumorzellennachweises im Knochenmark, insbesondere die eingesetzten Antikörper, wurden und werden in den Fachkreisen immer wieder diskutiert.

Treten DTCs sowohl bei prä- als auch bei postmenopausalen Patientinnen auf? Wo findet man sie häufiger?

In den mir bekannten Analysen treten DTCs unabhängig vom Menopausenstatus auf.

Gibt es DTCs auch schon bei kleinen Tumoren? Ja. Es handelt sich wie gesagt um einen unabhängigen Prognosefaktor, deshalb gibt es keine wirklich starke Korrelation zur Tumorgröße.

Zusammengefasst: Unabhängiger Prognosefaktor bedeutet also, dass es keine Korrelation zur Tumorgröße oder zum Menopausenstatus gibt? Genauso ist es. Man kann zwar beobachten, dass die Parameter etwas miteinander zu tun haben, aber sie beeinflussen sich nicht gegenseitig.

Kann man durch eine adjuvante Therapie (Chemo-, Strahlentherapie, endokrine Therapien) das Auftreten von DTCs verhindern?

Es gibt Untersuchungen bei Patientinnen, die eine neoadjuvante Chemotherapie erhalten haben [16]. Bei diesen Patientinnen hat man gesehen, dass sich DTCs nicht aufhalten lassen. Das heißt, in aller Regel sind zum Zeitpunkt der adjuvanten Therapie die Tumorzellen bereits verschleppt [17-19]. Nach der Tumorentfernung dürfte aufgrund des Fehlens des Tumors keine Tumorzellaussaat mehr stattfinden. Bei zirkulierenden Tumorzellen mag das etwas anders sein. Es gibt nur wenige Studien, die nachweisen, dass zirkulierende Tumorzellen tatsächlich auf ein frühes Rezidiv hinweisen, wie zum Beispiel die SUCCESS-
Studie [20] und das Poster von Frau Dr. KasimirBauer [15].

Sie haben selbst eine Studie bei DTC-positiven Patientinnen durchgeführt. Würden Sie diese kurz erläutern?

Wir sind davon ausgegangen, dass die Tumorzellen im Knochenmark ein bestimmtes Milieu brauchen, um proliferieren zu können. Wir waren und sind der Meinung, dass es insbesondere Wachstumsfaktoren aus dem Knochen sind, die einen negativen Einfluss auf die Metastasierung oder einen positiven Einfluss auf das Tumorwachstum haben können. Und wir vertreten die Ansicht, dass man durch eine Normalisierung des Knochenstoffwechsels, z.B. indem man Osteoprotektion mit Bisphosphonaten betreibt, einen hemmenden Effekt auf das Tumorzellwachstum und die Metastasierung erzielt. Das haben wir in Tierversuchen mehrfach nachgewiesen. Aus diesem Grund haben wir dann in den 1990er-Jahren auch erstmals eine Studie bei Patienten durchgeführt.

Und was ist bei dieser Studie herausgekommen? In unserer Studie und in einer Nachfolgestudie konnte gezeigt werden, dass durch den adjuvanten Einsatz von oralem Clodronat die Inzidenz ossärer und auch viszeraler Metastasen bei Patientinnen mit Mammakarzinom vermindert war [10, 11]. Die Rezidivrate wurde gesenkt und das Überleben verbessert. Das haben später mehrere andere Studien bestätigt, ebenfalls bei Patienten, die adjuvant Clodronat bekommen haben $[12,21]$. Allerdings waren das alles keine Studien, bei denen Tumorzellen im Knochenmark nachgewiesen wurden. Was natürlich nicht heißt, dass nicht auch Patientinnen dabei waren, die Tumorzellen im Knochenmark hatten. Das ist sogar sehr stark zu vermuten, aber nicht nachgewiesen worden.

Aufgrund Ihrer 1998 publizierten Daten wurde das Therapiekonzept der täglichen Gabe von 1040 mg/Tag Clodronat bei DTC-positiven Patientinnen in der Universitätsfrauenklinik Essen als Standard übernommen. Die in den folgenden Jahren gesammelten Daten wurden retrospektiv analysiert und hier in San Antonio vorgestellt. Was war das für Sie spannendste Ergebnis dieser Analyse?

Es ist hochinteressant, dass sich bei dieser retrospektiven Analyse Patientinnen mit Tumorzellen im Knochenmark und adjuvantem Clodronat von ihrer Prognose her ähnlich ver- 
hielten wie Patientinnen, die keine Tumorzellen im Knochenmark hatten. Daraus kann man schließen, dass der Effekt des Clodronats auf die Entstehung von Knochenmetastasen und anderen Metastasen zu einem günstigen Ergebnis bei diesen Patientinnen geführt hat.

Aktuell wird die Relevanz des Nachweises von zirkulierenden Tumorzellen (CTCs) auch in der primären Situation untersucht. Die Essener Daten zeigen unter anderem, dass bei den Frauen, die einen DTC-positiven Befund hatten, nur zu 19\% CTCs festgestellt werden konnten. Wie erklärt sich dieser Unterschied?

Der Nachweis von zirkulierenden Tumorzellen entspricht einer Momentaufnahme, wohinge- gen die Tumorzellen im Knochenmark schon seit längerem vorhanden sein können. Insofern ist es gut möglich, dass bei Patientinnen Tumorzellen im Knochenmark nachgewiesen werden, im Moment der Blutentnahme aber keine zirkulierenden Tumorzellen nachweisbar sind.

Ist der Nachweis von CTCs über das Blut nicht einfacher als der Nachweis von DTCs aus dem Knochenmark?

Wenn das Knochenmarksaspirat während der Operation zur Tumorentfernung gewonnen wird, stellt das für die Patientinnen keine zusätzliche Belastung dar. Und der Nachweis von DTCs ist aus den gerade genannten Gründen wesentlich aussagekräftiger.
Wie beurteilen Sie aus Ihrer eigenen Erfahrung das Nebenwirkungsspektrum von Clodronat im Therapiealltag, insbesondere auch bei lang andauernden Therapien?

Das Nebenwirkungsspektrum von Clodronat ist niedrig. Gelegentlich sehen wir Patientinnen, die aufgrund von gastrointestinalen Beschwerden die Therapie nicht durchführen können, aber das ist eine Prozentzahl zwischen 3 und 5\%. Prinzipiell ist die Therapie mit Clodronat gut verträglich. Man kann die Substanz über lange Zeiträume von 5 oder 6 Jahren geben. Sie führt nicht zu Kieferosteonekrosen, sie ist nicht nierentoxisch und sie verursacht keine Akutphasenreaktionen.

\section{Weitere Studien zur adjuvanten Bisphosphonat- therapie}

Weitere adjuvante Bisphosphonatstudien untersuchten verschiedene endokrine Therapien oder eine Chemotherapie in Kombination mit Zoledronat. Die finalen Ergebnisse der ABCSG-12-Studie zeigten, dass Zoledronat in Kombination mit Tamoxifen oder Anastrozol bei prämenopausalen Mammakarzinom-Patientinnen das DFS im Vergleich zur alleinigen endokrinen Therapie signifikant verbessert [22]. Die ZO-FASTStudie bestätigte diese Ergebnisse, auch hier verbesserte sich das DFS mit der Einnahme von Zoledronat [23]. In der AZURE-Studie zeigte sich im gesamten Studienkollektiv kein Vorteil durch die Hinzunahme von Zoledronat zur adjuvanten Standardtherapie hinsichtlich des DFS und des OS. Jedoch traten weniger Knochenmetastasen auf und postmenopausale Patientinnen profitierten [24].

\section{Fazit}

Bisphosphonate eignen sich nicht nur zur Therapie von Knochenmetastasen und zur Prävention von SREs und Hyperkalzämie. Eine Reihe von Studien belegt die Reduktion von Metastasen und einen Überlebensvorteil durch die adjuvante Bisphophonattherapie bei postmenopausalen Frauen oder mit nachgewiesenen DTCs. Die orale adjuvante Bisphosphonattherapie mit Clodronat ist dabei gut verträglich. Gegenüber der halbjährlichen Applikation eines intravenösen Bisphosphonats bietet die tägliche orale Gabe außerdem den Vorteil kontinuierlicher Wirkspiegel. Die AGO-Leitlinien 2015 empfehlen Clodronat oder Aminobisphosphonate bei postmenopausalen Patientinnen (www.ago-online.de/fileadmin/downloads/ leitlinien/mamma/maerz2015/de/2015D_21_Osteoonkologie_ und_Knochengesundheit.pdf). Auch prämenopausale Patientinnen unter ovarieller Suppression können von einer adjuvanten Bisphosphonattherapie profitieren.

Mascha Pömmerl, Feldkirchen-Westerham

\author{
Literatur \\ 1 Schindlbeck C et al.: Int J Gynecol 2007;17:1047-1055. \\ Páez D et al.: Clin Cancer Res 2012;18:645-653. \\ Fehm T et al.: APMIS 2008;116:742-753. \\ Mansi JL et al.: Lancet 1999;354:197-202. \\ Braun S et al: N Engl J Med 2000;342:525-533. \\ Hartkopf AD et al.: Eur J Cancer 2014;50:2550-2559. \\ Diel IJ et al.: J Natl Cancer Inst 1996;88:1652-1658. \\ Braun S et al.: N Engl J Med 2005;353:793-802. \\ 9 Diel IJ et al.: N Engl J Med 1998;339:357-363. \\ 10 Diel IJ et al.: Ann Oncol 2008;19:2007-2011. \\ 11 Powles T et al.: J Clin Oncol 2002;20:3219-3224. \\ 12 Paterson AH et al.: Lancet Oncol 2012;13:734-742. \\ 13 Coleman R et al.: Cancer Res 2013;72:abstr S4-07. \\ 14 Saarto T et al.: Acta Oncol 2004;43:650-656. \\ 15 Kasimir-Bauer S et al.: San Antonio Breast Cancer Symposium 2014,abstr P 04-01-07 \\ und Poster. \\ 16 Mathiesen RR et al.: Breast Cancer Res 2012;14:R117. \\ 17 Wiedswang G et al.: Clin Cancer Res 2004;10:5342-5348. \\ 18 Janni W et al.: Cancer 2005;103:884-891. \\ 19 Janni W et al.: Clin Cancer Res 2011;17:2967-2976. \\ 20 Rack B et al.: J Natl Cancer Inst 2014;106, doi: 10.1093/jnci/dju066. \\ 21 Powles T et al.: Breast Cancer Res 2006;8:R13. \\ 22 Gnant M et al.: Ann Oncol 2015;26:313-320. \\ 23 Coleman R et al.: Ann Oncol 2013;24:398-405. \\ 24 Coleman R et al.: Lancet Oncol 2014;15:997-1006.
}

\section{Impressum}

Können Bisphosphonate die Prognose von MammakarzinomPatientinnen mit DTCs im Knochenmark verbessern?

Beilage zu Breast CARE 10 | 2 | 15

Mit freundlicher Unterstützung durch

RIEMSER Pharma GmbH

Die Wiedergabe von Gebrauchsnamen, Handelsnamen, Warenbezeichnungen usw. in dieser Zeitschrift berechtigt auch ohne besondere Kennzeichnung nicht zur Annahme, dass solche Namen im Sinne der Warenzeichen- und Markenschutz-Gesetzgebung als frei zu betrachten wären und daher von jedermann benutzt werden dürfen. Für Angaben von Dosierungsanweisungen und Applikationsformen kann vom Verlag keine Haftung übernommen werden. Derartige Angaben müssen vom jeweiligen Anwender im Einzelfall anhand anderer Literaturstellen auf ihre Richtigkeit überprüft werden.

Ohne schriftliche Genehmigung des Verlags dürfen diese Publikation oder Teile daraus nicht in andere Sprachen übersetzt oder in irgendeiner Form mit mechanischen oder elektronischen Mitteln (einschließlich Fotokopie, Tonaufnahme oder Mikrokopie) reproduziert oder auf einem Datenträger oder Computersystem gespeichert werden. Alle Rechte vorbehalten.

() 2015 by S. Karger Verlag für Medizin und Naturwissenschaften GmbH, Postfach, 79095 Freiburg, Deutschland.

Druck: Kraft Druck GmbH, 76275 Ettlingen, Deutschland. 